\title{
The Algorithmic Design of The Line-tracking Car Based on The Positional PID
}

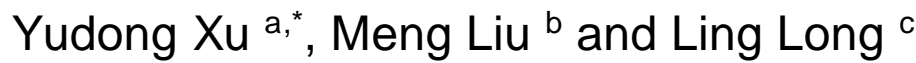 \\ College of Electric and Information Engineering, Southwest University for Nationalities, 610041, \\ Chengdu, china \\ a515704170@qq.com, b1792567679@qq.com, clong_ling_II@163.com
}

Corresponding author: Yudong $\mathrm{Xu}$

Keywords: Algorithmic Design, Line-tracking Car, Positional PID.

\begin{abstract}
In some applications, the intelligent car needs to walk along the wire. This paper presents a control algorithm for smart car to track the wire. We developed and implemented an algorithm on a smart car with a metal sensor, a single coil, a LDC1000. We conducted a number of inspection tests to verify the feasibility and effectiveness of the algorithm, and overcame the shortcomings that the traditional way is not smooth.
\end{abstract}

\section{Introduction}

The smart car is running on a runway that have a metal line, the diameter of the wire is 0.6 to $0.9 \mathrm{~mm}$. Compared with the traditional black tape, the wire is less affected by the ambient light and has strong anti-interference ability. Even in the dark environment can also complete the tracking task, wire-tracking has been applied in the warehouse transportation robot. This paper studies the algorithm of the intelligent car tracking the metal line. We used the high-performance controller STM32 F407ZGT6 as the control core. The platform structure is that the two wheels in the front and one wheel in the back, the wheels in the front using the DC motor to control the swerve, the wheels behind using the universal wheels.

\section{The Principle of Position Digital PID Algorithm}

PID and its derived algorithms are the most widely used algorithms in industrial applications. They are well-deserved universal algorithms and are widely used in a variety of mechanical devices (such as vehicles, robots, rockets). The general form of the PID algorithm is shown in Figure 2.1.

PID controller is a linear regulator that it combines the proportion (P), integral (I) and differential (D) of the deviation, which between the given value and the actual value, by a linear combination to constitute the control volume, and to control the object. The differential equation of the PID regulator is that

$$
u(t)=K_{p} e(t)+K_{i} \int_{0}^{t} e(t) d t+K_{d} \frac{d e(t)}{d t}
$$

In the equation, $u(t)$ is the PID output value, $e(t)$ is the difference between the expected value and the actual value, $\int_{0}^{t} e(t) d t$ is the integral value, $d e(t) / d t$ is the differential value, $K_{p}$ is the proportional coefficient, $K_{i}$ is the integral coefficient, and $K_{d}$ is the differential coefficient. Then discretized the differential amount and the integral amount to obtain the PID calculation epution. In this equation, $T$ is the sampling time.

$$
u(k)=K_{p} e(k)+K_{i} \sum_{i=0}^{k} e(i) T+K_{d} \frac{e(k)-e(k-1)}{T}
$$




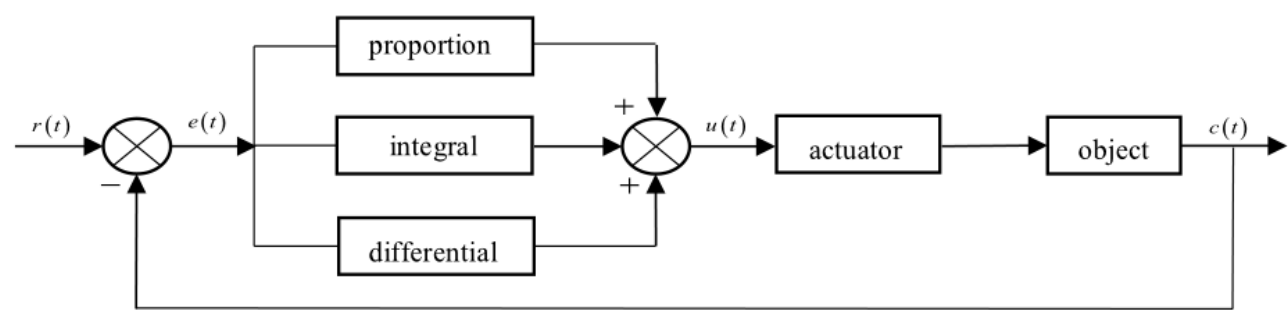

Figure 2.1 PID Regulator Principle Block Diagram

\section{The Line-Tracking Algorithm Design of The Car}

\subsection{The Path Detection Strategy Based on PID Algorithm}

Using the PID algorithm, this paper processed the path detection information, controlled the direction of the car. And both achieved a better control effects.

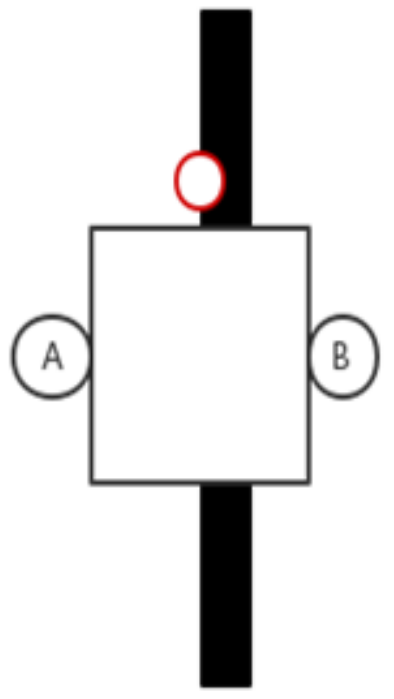

Figure 3.1.1 The Car Running Diagram.

As shown in Figure 3.1.1, the rectangular refers to the car, the black part is the wire, A and B are two wheels, the front of the car is equipped with a vertically downward LDC1000 metal sensor, the red circle is the range that the sensor could detect, also means the coil, and the coil on the top of the wire's edge. The arrow is the direction of the car.

Because the sensor in the wire above the data collected around 6000 or so, the sensor completely deviates from the wire when the data collected is about 3000, that is, the sensor from the top of the wire deviation from the bottom did not detect the wire, the collection of the value of the back from large to small gradient changes.

The value of the line is divided into two parts, the car turn right when the LDC1000 sensor value is less than 4500, the car turn left when the sensor value is greater than 4500 , the car straight when the sensor value is equal to 4500. As shown in Figure 3.1.2. Although this method can also complete the car line patrol task, but the car will not be too fast, and the car will be shaking, it is looks very bad. Even if the wire is straight, this method can't make the car straight. In order to make the linetracking smoother, we introduced a three-stage line inspection method.

The sensor reading is divided into three sections, as shown in Figure 3.1.3. The car turn right when the sensor readings less than 3500, the car straight when the sensor reading value of 3500-5500, the car turn left when the sensor readings greater than 5500. The effect of the second way is better than the first way. At least the car will go straight ahead. As with the first method, it is still necessary to determine which steering mode to use (small or obvious steering action) based on the characteristics of the sensor. The car will still have a considerable number of swing back and forth, so that the car greatly reduced the speed. 

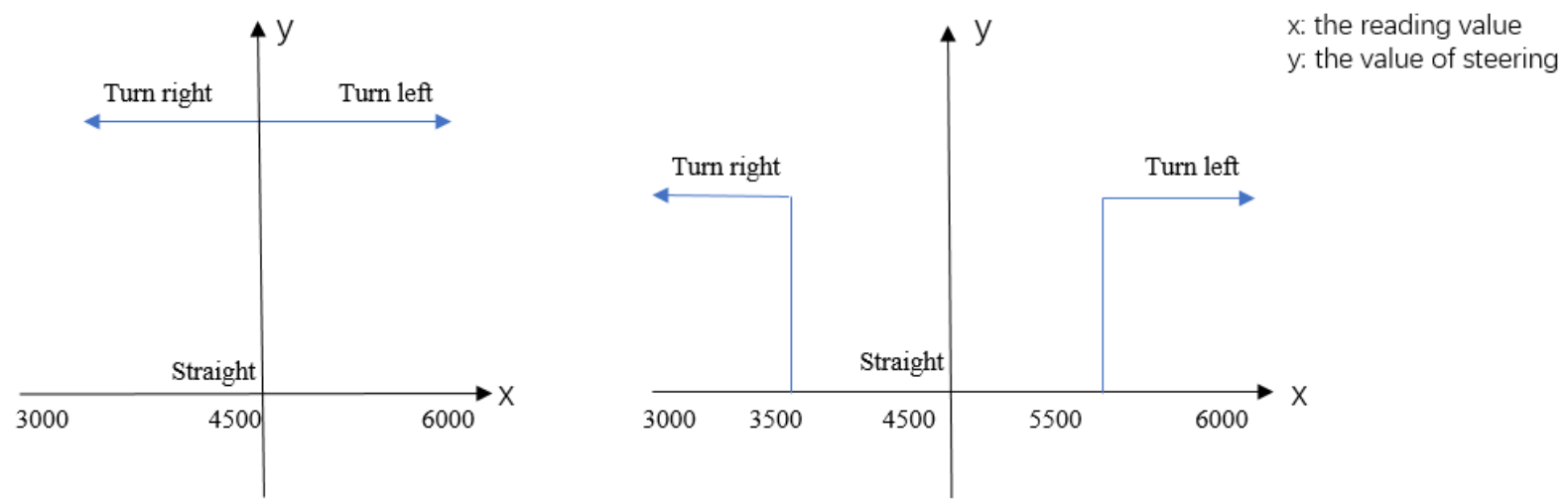

Figure 3.1.2. The Line-tracking of Two-stage Figure 3.1.3. The Line-tracking of There-stage

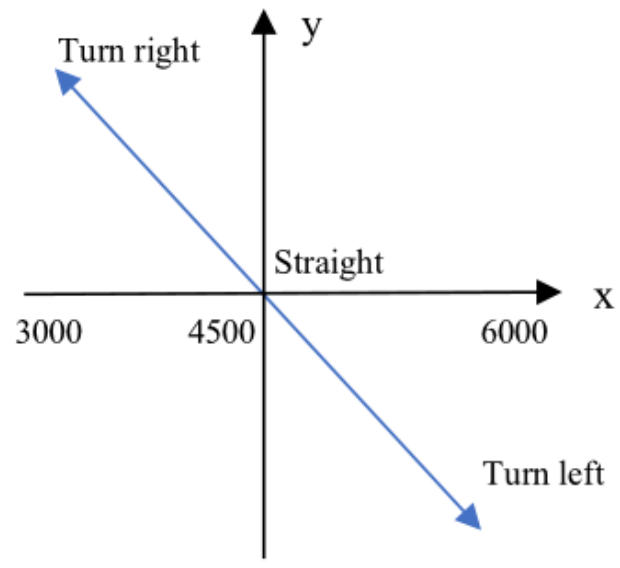

Figure 3.1.4. The Line-tracking of Proportional Control

It can be seen, when the sensor readings are divided into infinite, that is, in the program to establish an infinite car steering strategy, the car control effect will be significantly better. As shown in Figure 3.1.4, this is a proportional control patrol car, the change between the two limit points is very smooth. If the reading value of the sensor indicates that the car is close to the wire, the small car makes a small turn. if the reading value indicates that the car is very far from the wire, the small car makes a big turn. This is actually the proportion of PID algorithm links, the proportion is an important concept. The meaning of the ratio is that there is a linear relationship between the two variables, simply say that the relationship between the variables is presented as a straight line.

\subsection{The Steering Control Based on PID Algorithm}

Based on equation (2), we obtained the position control algorithm

$$
\text { Position_out }(k)=K_{p} e(k)+K_{i} \sum_{i=0}^{k} e(i) T+K_{d} \frac{e(k)-e(k-1)}{T}
$$

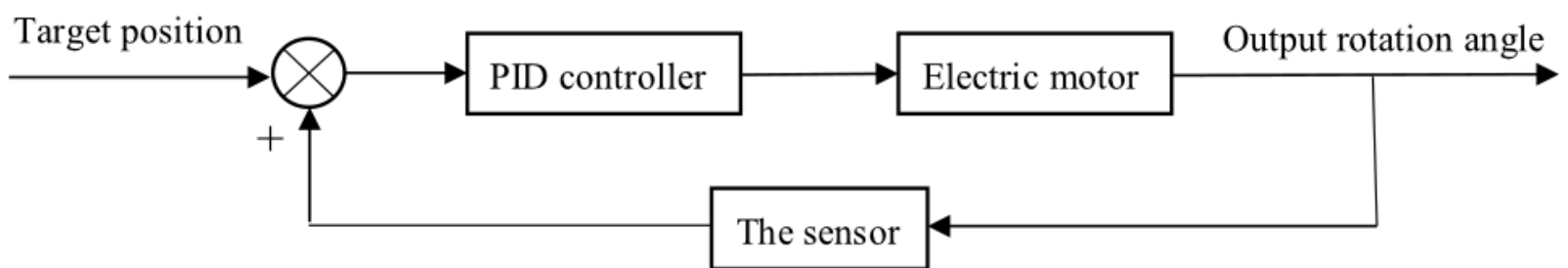

Figure 3.2.1. Block Diagram of PID Control

This equation is the formula of positional PID. Position_out $(K)$ is the output of the position loop, $e(K)$ is equal to the car's target position minus the actual position. The block diagram of the PID control is shown in the figure. 


\section{Program Implementation}

The program block diagram of the PID control is shown in Figure 4.1.

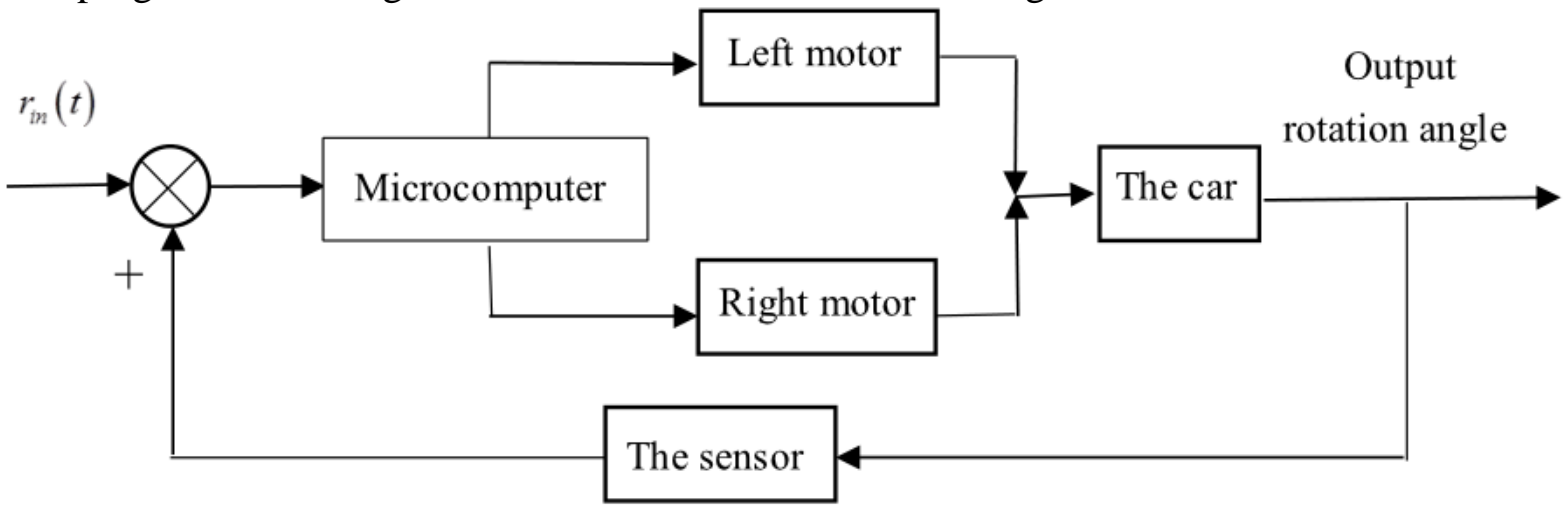

Figure 4.1 Block Diagram of PID Control

$r_{i n}(t)$ is the value of the sensor when the cart is straight, about 4500 . Define a target power value POINT for the car, this value also is the PWM value of the motor when the cart is straight. The output angle Position_out $(K)$ is calculated from equation (3), than the PWM value of the left and right motor are POWERA $=$ POINT + Position_out $(K)$, and POWERB $=$ POINT - Position_out $(K)$. From the equation (3), we can see that $e(K)$ is greater than 0 and Position_out $(K)$ is also greater than 0 when the car is turn left from the edge of the wire. At this point, POWERA will increase and turn the car to the right. $e(K)$ is smaller than 0 and Position_out $(K)$ is also smaller than 0 when the car is turn right from the edge of the wire. At this point, POWERA will decrease and turn the car to the left.

When the car isn't always smooth along the wire walking, we can add the integral to PID controller. The integral reflects the cumulative deviation of the system, which can make the system eliminate the steady-state error. Because of the error, the integral adjustment is carried out until there is no error. An important role of integral control is to be able to remove small errors. If the sensor is very close to the edge of the line, but not just on the edge of the line, then $\mathrm{e}(\mathrm{K})$ will be very small, it can only produce a small amount of correction. The integral control part can be a perfect correction of small errors. When the stability of the car is required to be higher, it should be added to the differential part of the PID controller. The derivative is actually the derivative of the higher mathematics. The error change between the two successive points is called the derivative. The derivative is a straight slope. It can predict the steering change of the car in advance, the principle is that the expected value of the next error is equal to the current error plus the first two changes in the sampling error of the sensor.

\section{Parameter Setting}

In order to make the trolley smoothly along the wire, it is necessary to set the proportional parameter $K p$, the integral parameter $\mathrm{Ki}$ and the derivative parameter $K d$ of the PID formula.

From the control law of each parameter, the proportional parameter $K p$ makes the reaction fast, the differential parameter $K d$ makes the reaction advance, and the integral parameter $K i$ lags the reaction. Within a certain range, the greater the value of $K p, K d$, the better the effect of the adjustment. The adjustment principle of each parameter is as follows:

When the output is not oscillating, increase the proportional gain $K p$. When the proportional gain $K p$ is determined, the integral term and the derivative term of the PID are removed first, usually order $\mathrm{Ki}=0, K d=0$, PID is the pure proportional adjustment. The input is set to $60 \%$ to $70 \%$ of the maximum value allowed by the system, and the proportional gain $K p$ is gradually increased from 0 until the system oscillates; In turn, the proportional gain $K p$ is gradually reduced from this time until the system oscillation disappears, and the proportional gain $K p$ at this time is recorded. The proportional gain $K p$ of the PID is set to $60 \%$ to $70 \%$ of the current value. Proportional gain $K p$ debugging is complete. 
When the output is not oscillating, decrease the integration time constant $\mathrm{Ti}$. After the proportional gain $K p$ is determined, the initial value of a larger integral time constant $K i$ is set and then gradually decreases $K i$ until the system oscillates, and then, in turn, gradually increases $K i$ until the system oscillation disappears. Record the $K i$ at this time, set the PID integration time constant $K i$ for the current value of $150 \%$ to $180 \%$. The integral time constant $K i$ is completed.

When the output is not oscillating, increase the derivative time constant $K d$. As with the method of determining $K p$ and $K i, K d$ takes $30 \%$ of the oscillation.

\section{Acknowledgements}

This work was financially supported by the Fundamental Research Funds for the Central Universities of Southwest University for Nationalities (NO. 2016NZYQN08).

\section{References}

[1] Jianwei Ma, Satisfied PID control design theory and method, Science Press, 2007,1.

[2] Jinkun Liu, Advanced PID control MATLAB - simulation, Electronic Industry Press, 2004, 9.

[3] National College Students Electronic Design Competition winning works selected, the National College Students Electronic Design Competition Organizing Committee (1994 1995) [M]. Beijing: Beijing Polytechnic University Press, 1997 first edition.

[4] Shousong Hu, Basic Principles of Automatic Control Course (3rd Edition), Science Press, March 2013.

[5] Zehua Zhang, Xiaolei Wang, Leilei Zhang, Design of Wall Wire Position Measuring Instrument Based on LDC1000 Inductance Digital Sensor, Electronic Design Engineering Press, 2015.

[6] Haoqiang Tan, C language programming [M]. Beijing: Tsinghua University Press, 2012. 